\title{
Combination of Acellular Nerve Graft and Schwann Cells-Like Cells for Rat Sciatic Nerve Regeneration
}

\author{
Songtao Gao, ${ }^{1}$ Yan Zheng, ${ }^{2}$ Qiqing Cai, ${ }^{1}$ Zhansheng Deng, ${ }^{3}$ Weitao Yao, \\ Jiaqiang Wang, ${ }^{1}$ Xin Wang, ${ }^{1}$ and Peng Zhang ${ }^{1}$ \\ ${ }^{1}$ Department of Orthopedics, The Affiliated Tumor Hospital of Zhengzhou University, No. 127, Dongming Road, \\ Zhengzhou, Henan 450008, China \\ ${ }^{2}$ Department of Radiology, The First Affiliated Hospital of Zhengzhou University, No. 1, Jianshe Road, \\ Zhengzhou, Henan 450052, China \\ ${ }^{3}$ Department of Orthopedics, Xiangya Hospital of Central South University, No. 87, Xiangya Road, \\ Changsha, Hunan 410008, China \\ Correspondence should be addressed to Qiqing Cai; hnszlyygk@163.com
}

Received 29 March 2014; Revised 28 May 2014; Accepted 16 June 2014; Published 9 July 2014

Academic Editor: Leszek Kaczmarek

Copyright (C) 2014 Songtao Gao et al. This is an open access article distributed under the Creative Commons Attribution License, which permits unrestricted use, distribution, and reproduction in any medium, provided the original work is properly cited.

Objective. To investigate the effect of tissue engineering nerve on repair of rat sciatic nerve defect. Methods. Forty-five rats with defective sciatic nerve were randomly divided into three groups. Rats in group A were repaired by acellular nerve grafts only. Rats in group B were repaired by tissue engineering nerve. In group C, rats were repaired by autogenous nerve grafts. After six and twelve weeks, sciatic nerve functional index (SFI), neural electrophysiology (NEP), histological and transmission electron microscope observation, recovery ratio of wet weight of gastrocnemius muscle, regenerated myelinated nerve fibers number, nerve fiber diameter, and thickness of the myelin sheath were measured to assess the effect. Results. After six and twelve weeks, the recovery ratio of SFI and wet weight of gastrocnemius muscle, NEP, and the result of regenerated myelinated nerve fibers in groups B and C were superior to that of group A $(P<0.05)$, and the difference between groups B and $\mathrm{C}$ was not statistically significant $(P>0.05)$. Conclusion. The tissue engineering nerve composed of acellular allogenic nerve scaffold and Schwann cells-like cells can effectively repair the nerve defect in rats and its effect was similar to that of the autogenous nerve grafts.

\section{Introduction}

Peripheral nerve injury is a common disease, the proliferation of glia and extraneural connective tissue in peripheral nerve defect may hinder the growth of anagenetic axon or misguide the growth cone of axon; thus, the anagenetic axon cannot reach the target organ or even results in the formation of neuroma at the nerve stumps [1]; therefore, the defect of nerve must be bridged by graft to induce the nerve regeneration. As the golden standard of the peripheral nerve defects therapy, the autologous nerve graft was effective for nerve repair, but the source of autologous nerve graft was limited and the application of this graft was limited by several elements such as the injury to donor for graft acquisition and the limited length for nerve defect repairment [2]. Other methods, such as nerve allograft, nonnerve tissue graft, and artificial nerve tissue graft, had been used, but their effect was not satisfactory [3].

This research intended to use the Schwann cells-like cells that differentiated from Adipose derived stem cells (ADSCs) as the seed cells and acellular nerve graft processed by chemical method as the three-dimensional cells scaffolds to construct the tissue-engineering peripheral nerve graft which used to repair the sciatic nerve defect of rats, thus providing the experimental base for the clinical application.

\section{Materials and Methods}

2.1. Animals. Twenty-five Sprague-Dawley rats and sixty $\mathrm{F}_{344}$ inbred rats (provided by Animal Experimental Center of Zhengzhou University) weighing around $200 \mathrm{~g}$ were used; all 
animals utilized in this research were cared for according to the policies and principles established by the animal welfare act and the NIH guide for the care and use of laboratory animals.

\subsection{Methods}

2.2.1. Preparation of Acellular Nerve Scaffold. Twenty-five Sprague-Dawley rats were sacrificed by intraperitoneal anesthesia with $10 \%$ chloral hydrate solution $(0.5 \mathrm{~mL} / 100 \mathrm{~g})$. After immersion sterilized in $75 \%$ alcohol for $15 \mathrm{~min}$, bilateral sciatic nerves of $20 \mathrm{~mm}$ (total 50 nerves) were harvested for experiment under aseptic conditions. The isolated sciatic nerves were cleaned of external debris under a LZL-21 dissecting microscopy (Zhenjiang, Jiangsu, China). All nerves were used to prepare the acellular nerve graft according to Hudson method [4].

2.2.2. Culture and Inducing Differentiation of ADSCs. Fifteen $\mathrm{F}_{344}$ inbred rats were sacrificed by intraperitoneal anesthesia with $10 \%$ chloral hydrate solution $(0.5 \mathrm{~mL} / 100 \mathrm{~g})$. After immersion sterilized in $75 \%$ alcohol for $15 \mathrm{~min}$, bilateral inguinal fat pad was harvested for experiment under aseptic conditions and minced after washing by PBS (phosphate buffer solution) and then dissociated with $0.075 \%$ collagenase type I for $90 \mathrm{~min}$; the solution was passed through a $75 \mu \mathrm{m}$ filter to remove undissociated tissue and then neutralized by the DMEM of low glucose that contains $20 \%$ (v/v) fetal bovine serum and centrifuged at $1000 \times \mathrm{g}$ for $8 \mathrm{~min}$. The stromal cell pellets were resuspended in DMEM of low glucose that contains $20 \%(\mathrm{v} / \mathrm{v})$ fetal bovine serum with $1 \%(\mathrm{v} / \mathrm{v})$ penicillin/streptomycin solution, inoculated in $25 \mathrm{~mL}$ culture bottles in a density of $4 \times 10^{5} / \mathrm{mL}$. The medium was replaced after 3 4 d, and the nonadherent cells were removed. The cells were passaged with trypsin/EDTA and inoculated in $50 \mathrm{~mL}$ culture bottles when the ratio of cells fusion was up to $90 \%$. Cultures were maintained in a $37^{\circ} \mathrm{C}$ incubator with $5 \% \mathrm{CO}_{2}$; the fourth generation cells were induced to differentiation [5]. The adipose derived stem cells under subfused status of the fourth generation were used for induction according to Dezawa method [6] that induces the bone marrow stromal stem cells (BMSCs) to differentiate into Schwann-like cells in vitro. On the fifth day after induction, the ADSCs were digested using $0.25 \%$ trypsin and $0.02 \%$ EDTA and dropped in aseptic glass slides that were coated with polylysine after centrifugation and collection. Then the cells were incubated in $37^{\circ} \mathrm{C}$ for $2 \mathrm{~h}$, washed by PBS for $5 \mathrm{~min}$, dried and fixed by $4 \%$ paraformaldehyde, and stained by S-100 and glial fibrillary acidic protein (GFAP).

2.2.3. Animal Experiment. Forty-five $\mathrm{F}_{344}$ inbred rats were randomly divided into three groups according to different methods of sciatic nerve defect repair; there were fifteen rats in each group. After intraperitoneal anesthesia with $10 \%$ chloral hydrate solution $(0.5 \mathrm{~mL} / 100 \mathrm{~g})$; the rats were placed in a prone position and the skin was prepared. A standard longitudinal incision was made in the left gluteal region, and then the tissue between the subcutaneous and muscular layers was dissected. Following this, the main stem of left sciatic nerve was isolated at midthigh level and $10 \mathrm{~mm}$ nerve was removed under aseptic conditions; the nerve defect was repaired with different methods: group $A$, nerve defect was repaired using acellular nerve graft alone; group $B$, nerve defect was repaired with acellular nerve graft and Schwann cells-like cells that differentiated from ADSCs: when the ratio of cells fusion was up to $50 \%$, the culture medium was replaced by $10 \mu \mathrm{mol} / \mathrm{mL}$ culture medium that labeled by BrdU (5-bromo-2-deoxyuridine), a cell proliferation marker, cultures were maintained in a $37^{\circ} \mathrm{C}$ incubator with $5 \% \mathrm{CO}_{2}$ for $24 \mathrm{~h}, 200 \mu \mathrm{L}$ Schwann cells-like cells that differentiated from ADSCs were suspended in Dulbecco's Modified Eagle Medium (DMEM) with $10 \%$ fetal bovine serum in a density of $1 \times 10^{7} / \mathrm{mL}$, and then $10 \mu \mathrm{L}$ cells suspension were pointinjected into the $10 \mathrm{~mm}$ acellular nerve grafts by microinjector from one end to the other through the central axis of graft's cavity. After $4 \mathrm{~h}$, the cells-scaffold combinations were placed in plates containing DMEM with $10 \%$ fetal bovine serum for $48 \mathrm{~h}$ at $37^{\circ} \mathrm{C}$ and $5 \% \mathrm{CO}_{2}$ under saturated humidity for use; Group $\mathrm{C}$, nerve defect was repaired with autogenous nerve that suture in situ. All the grafts were end-to-end anastomosis with the two ends of sciatic nerve defect by $9 / 0$ nontraumatic suture needles and the normal right sciatic nerves were used as control.

(1) Recovery Ratio of Sciatic Nerve Function Index (SFI\%). The SFI was calculated using the method described by Reynolds and Weiss [7]. At $6 \mathrm{w}$ and $12 \mathrm{w}$ following the surgery, footprints' record box made by us was used to measure the following variable quantity. The box was $8.5 \mathrm{~cm}$ wide and $50 \mathrm{~cm}$ long, with one door on the side of the box and a piece of $70 \mathrm{~g}$ white paper that is same size as the bottom of the box was placed in the bottom of the box. Rats that two hind paws had been dipped in carbon black ink walked from one end to the side door of the box, and three or four footprints of each foot were left on the paper. Three variable quantities of the experimental side feet and the normal side feet were measured as follows.

(1) Print length (PL): the maximum print distance between heel to toe, the accuracy of length in millimeters and every time the maximum PL was used.

(2) Toe spread (TS): the distance between 1st toe and 5th toe, the accuracy of length in millimeters and every time the maximum TS was used.

(3) Intermediary toe spread (ITS): the distance between 2nd toe and 4th toe, the accuracy of length in millimeters and every time the maximum ITS was used.

The SFI was calculated in accordance with the formula described by Bain et al. [8] to work out SFI:

$$
\begin{aligned}
\mathrm{SFI}= & -38.3\left(\frac{\mathrm{EPL}-\mathrm{NPL}}{\mathrm{NPL}}\right)+109.5\left(\frac{\mathrm{ETS}-\mathrm{NTS}}{\mathrm{NTS}}\right) \\
& +13.3\left(\frac{\mathrm{EIT}-\mathrm{NIT}}{\mathrm{NIT}}\right)-8.8
\end{aligned}
$$


$\mathrm{SFI}=0$ was used as normal value, $\mathrm{SFI}=-100$ was used as complete nerve transected, and recovery ratio of sciatic nerve function index (SFI\%) was worked out by comparing the SFI of experimental side to that of the normal side.

(2) Neural Electrophysiology (NEP). $6 \mathrm{w}$ and $12 \mathrm{w}$ following the surgery, BL-410 biological function experiment system and related equipment were used to mensurate the motor nerve conduction velocity (MNCV) of bilateral sciatic nerve according to Foidart-Dessalle method [9] and the maximum amplitude of complex action potential (CMAP) of bilateral sciatic nerve, so the recovery ratio of them was calculated according to the following formulae:

$$
\begin{aligned}
& \mathrm{MNCV}(\mathrm{ms})=\frac{\text { Distance between two points }(\mathrm{m})}{\text { Difference value in latent period of two ends }(\mathrm{s})}, \\
& \begin{array}{r}
\text { Recovery ratio of } \mathrm{MNCV}=\frac{\text { the } \mathrm{mncv} \text { in operated side }}{\text { the } \mathrm{mncv} \text { in normal control side }} \\
\times 100 \%,
\end{array} \\
& \begin{array}{r}
\text { Recovery ratio of CMAP } \\
=\frac{\text { the mean amplitude in operated side at different times }}{\text { the mean amplitude in normal control side at different times }} \\
\quad \times 100 \% .
\end{array}
\end{aligned}
$$

(3) Recovery Ratio of Wet Weight of Gastrocnemius Muscle. After the neural electrophysiology experiment was finished, the gastrocnemius muscle of experiment and normal legs in all rats were taken out completely, and the wet weight of gastrocnemius muscle was weighed by electronic balance such that precision is $1 / 10000$ grams. The recovery ratio of weight of gastrocnemius muscle was worked out by comparing the weight of gastrocnemius muscle of experimental side to that of the normal side.

(4) Histological Observation. After the above experiment, $3 \mathrm{~mm}$ nerve segment in the proximal part of nerve grafts and the parallel segment in the control nerve were fixed by $10 \%$ formalin fixation for $24 \mathrm{~h}$; gradient alcohol dehydration and paraffin embedded, transverse, and longitudinal sections $(5 \mu \mathrm{m})$ of them were cut with a microtome. The tissue slices were deparaffinized with xylene $2 \times 10 \mathrm{~min}$ and then rinsed in anhydrous alcohol $2 \times 2 \mathrm{~min}$ and immersed in 95\%, $80 \%$, and $75 \%$ alcohol $1 \mathrm{~min}$, respectively, and then rinsed in PBS $1 \mathrm{~min}$. After staining by Harris hematoxylin for $5 \mathrm{~min}$, the slices were rinsed in PBS for $1 \mathrm{~min}$ and differentiated with $10 \mathrm{~mL} / \mathrm{L}$ hydrochloric acid alcohol $20 \mathrm{~s}$. After rinsed in PBS for $1 \mathrm{~min}$ and returned to blue with $10 \mathrm{~mL} / \mathrm{L}$ ammonia for $30 \mathrm{~s}$, then rinsed in PBS for $1 \mathrm{~min}$. After incubation in eosin staining $1 \mathrm{~min}$, the slices were rinsed in PBS $30 \mathrm{~s}$ and dehydrated in $75 \%$ alcohol $20 \mathrm{~s}$, in $80 \%$ alcohol $30 \mathrm{~s}$, and in $95 \%$ alcohol $2 \times 1 \mathrm{~min}$. After rinsing in anhydrous alcohol 2 $\times 2 \mathrm{~min}$ and immersed in xylene $3 \times 2 \mathrm{~min}$, the slices were covered with general clarity gum. The longitudinal tissue slices from proximal part of nerve grafts in group B were deparaffinized with xylene $2 \times 10 \mathrm{~min}$ and incubated by $3 \%$ $\mathrm{H}_{2} \mathrm{O}_{2}\left(30 \% \mathrm{H}_{2} \mathrm{O}_{2}\right.$ mixed with methyl alcohol in ratio of $\left.1: 10\right)$ for $10 \mathrm{~min}$; after antigen repaired by microwave heating in citrate buffer solution, then the sealing liquid of 5\% BSA was added and the first antigen (rabbit anti-rat antigen of $\mathrm{BrdU}$ and S-100 protein) was added under $37^{\circ} \mathrm{C}$ for $90 \mathrm{~min}$, rinsed in PBS $2 \times 2 \mathrm{~min}$ and the biotin-labeled secondary antibody (goat anti-rabbit antigen) was added that incubated under the room temperature for $20 \mathrm{~min}$; $S A B C$ reagent was added and incubated under the room temperature for $20 \mathrm{~min}$, and 3,3-diaminobenzidine (DAB) was used as ingrain agent. Myelinated fibers and myelin sheaths of regenerated nerve were observed under a CX31-12C04 light microscopy (Olympus, Japan). $3 \mathrm{~mm}$ nerve segment in the middle part of nerve graft and the parallel segment in the control nerve were taken to fix in $4 \%$ glutaraldehyde in PBS for $4 \mathrm{~h}$ at room temperature, then rinsed in PBS $3 \times 3 \mathrm{~min}$, fixed in osmic acid, rinsed in PBS $3 \times 3 \mathrm{~min}$, and dehydrated in ascending series of alcohol (50\%, 70\%, 90\%, and 100\%); Epon 812 embedded, semithin section of $1 \mu \mathrm{m}$ was toluidine blue stained.

(5) Transmission Electron Microscope. $6 \mathrm{w}$ and $12 \mathrm{w}$ following the surgery, $3 \mathrm{~mm}$ nerve segments in the distal part of nerve grafts and the parallel segments in the control nerves were taken to fix in $4 \%$ glutaraldehyde in PBS for $4 \mathrm{~h}$ at room temperature, then rinsed in PBS $3 \times 3 \mathrm{~min}$, fixed in osmic acid, rinsed in PBS $3 \times 3 \mathrm{~min}$, and dehydrated in ascending series of alcohol (50\%, 70\%, 90\%, and 100\%); Epon 812 embedded, ultrathin section of $20 \mathrm{~nm}$ was uranyl acetate and lead citrate stained; the regeneration of nerve was observed under transmission electron microscope.

(6) Recovery Ratio of Quantity, Diameter, and Myelin Sheath Thickness in Regenerated Myelinated Nerve Fibers. $6 \mathrm{w}$ and $12 \mathrm{w}$ following the surgery, the toluidine blue staining slices of bilateral nerve segments in each group were observed under light microscope that magnified 1000 times to count the quantity of myelinated nerve fibers in each field of vision and contrast the quantity of experimental side to the quantity of normal side to get the recovery ratio:

Recovery ratio of the quantity of nerve fiber

$$
\begin{aligned}
= & \frac{\text { Nerve fiber quantity of the experimental side }}{\text { Nerve fiber quantity of the normal side }} \\
& \times 100 \%
\end{aligned}
$$

Both the diameter and myelin sheath thickness in regenerated myelinated nerve fibers of each group were assessed in a double blind method with MOTICMED 6.0 digital medical imagine analysis system by pathological technician in our laboratory: the section of bilateral nerve segment in each group was observed under light microscope that magnified 400 times to count the quantity of myelinated nerve fibers in four fields of vision and record the diameter and myelin sheath thickness; the average was counted and the quantity of experimental side was contrasted to the quantity of normal side to get the recovery ratio:

Recovery ratio of the diameter of nerve fiber

$$
\begin{aligned}
= & \frac{\text { Nerve fiber diameter of the experimental side }}{\text { Nerve fiber diameter of the normal side }} \\
& \times 100 \%,
\end{aligned}
$$




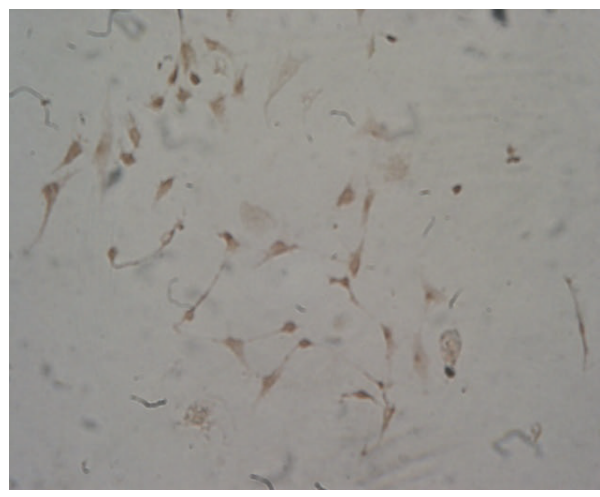

(a)

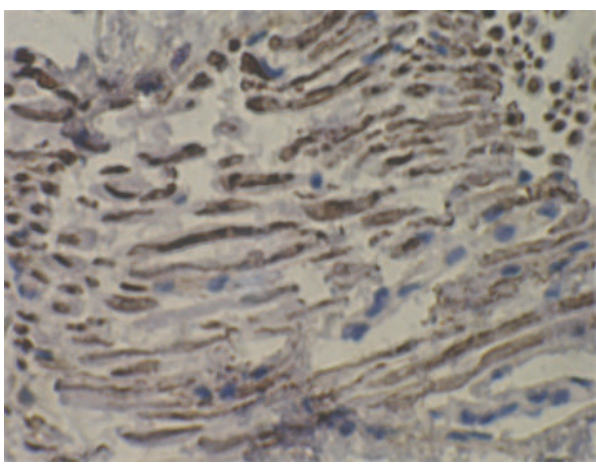

(c)

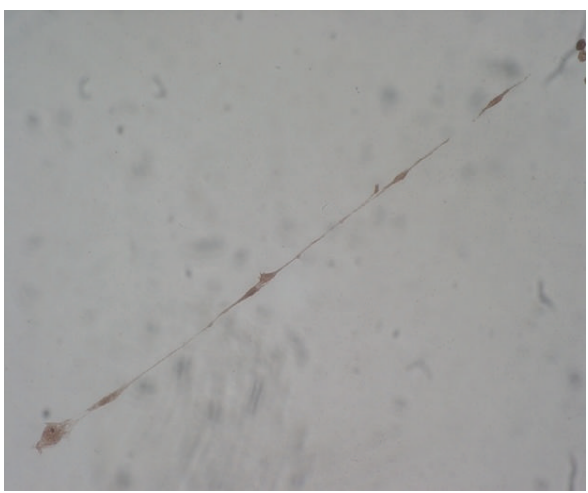

(b)

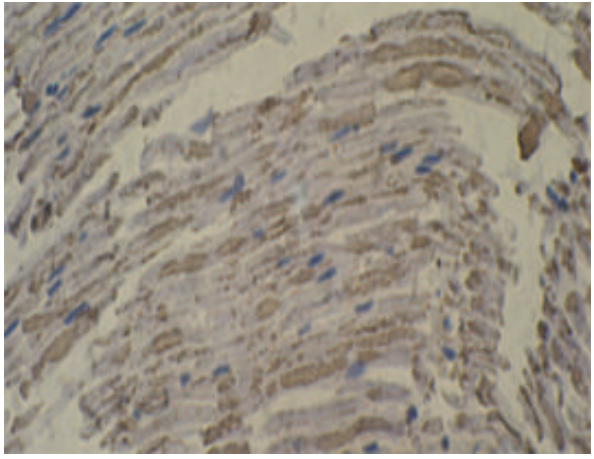

(d)

FIGURE 1: (a) Immunocytochemical staining of GFAP in Schwann cells-like cells that differentiated from ADSCs. (b) Immunocytochemical staining of S-100 in Schwann cells-like cells that differentiated from ADSCs. (c) BrdU and S-100 staining of longitudinal nerve slices from group B at $6 \mathrm{w}$, blue BrdU-labeled Schwann cells-like cells' nucleus, and brown S100-labeled staining myelin sheath were observed under microscope. (d) BrdU and S-100 staining of longitudinal nerve slices from group B at $12 \mathrm{w}$, blue BrdU-labeled Schwann cells-like cells' nucleus, and brown S100-labeled staining myelin sheath were observed under microscope.

Recovery ratio of the myelin sheath thickness of nerve fiber

$$
=\frac{\text { Thickness of the experimental side }}{\text { Thickness of the normal side }} \times 100 \% \text {. }
$$

2.3. Statistical Analysis. The results were analyzed by spss 10.0 (SPSS Inc., Chicago, IL, USA); the main statistical methods include one-way analysis of variance (ANOVA) which was performed to determine the statistical significance between groups, and $t$-tests were used to determine whether the averages of the data sets were statistically significant. Values of $P<0.05$ were considered to indicate a statistically significant.

\section{Results}

3.1. Identification of Schwann Cells-Like Cells That Differentiated from ADSCs. After staining by S-100 and GFAP, the cytoplasm of the positive staining cells was yellow dyed; the morphology of positive staining cells was consistent with that of living cells observed under inverted microscope (Figures 1(a) and $1(\mathrm{~b})$ ).

3.2. Recovery Ratio of Sciatic Nerve Function Index (SFI\%). $6 \mathrm{w}$ and $12 \mathrm{w}$ following the surgery, the SFI\% of groups B

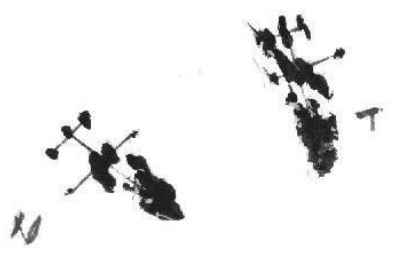

FIgURE 2: The footprint for sciatic nerve function index of rats.

and $\mathrm{C}$ was superior to that of group $\mathrm{A}(P<0.05)$, but the difference between groups $\mathrm{B}$ and $\mathrm{C}$ was not statistically significant $(P>0.05)$ (Table 1, Figure 2).

3.3. Neural Electrophysiology (NEP). $6 \mathrm{w}$ and $12 \mathrm{w}$ following the surgery, the recovery ratio of $\mathrm{MNCV}$ and CMAP of groups $\mathrm{B}$ and $\mathrm{C}$ was superior to that of group $\mathrm{A}(P<0.05)$, but the difference between groups $B$ and $C$ was not statistically significant $(P>0.05)$ (Table 1, Figure 3$)$.

3.4. Histological Observation. $6 \mathrm{w}$ following the surgery, in $\mathrm{HE}$ staining slices of three groups, the regenerated myelinated 
TABLE 1: Comparison of SFI\%, MNCV\%, and CAMP\% in 3 groups $(\bar{x} \pm s, n=15)$.

\begin{tabular}{lcccccc}
\hline & \multicolumn{2}{c}{ SFI\% } & \multicolumn{2}{c}{ MNCV\% } & \multicolumn{2}{c}{ CAMP\% } \\
& $6 \mathrm{w}$ & $12 \mathrm{w}$ & $6 \mathrm{w}$ & $12 \mathrm{w}$ & $6 \mathrm{w}$ & $12 \mathrm{w}$ \\
\hline Group A & $46.32 \pm 5.13^{*}$ & $72.73 \pm 8.06^{*}$ & $42.54 \pm 6.33^{*}$ & $69.34 \pm 9.13^{*}$ & $40.52 \pm 4.15^{*}$ & $68.24 \pm 9.45$ \\
Group B & $52.38 \pm 7.12^{\#}$ & $79.99 \pm 10.33^{\#}$ & $51.12 \pm 8.15^{\#}$ & $75.93 \pm 5.95^{\#}$ & $45.38 \pm 4.12^{\#}$ & $75.98 \pm 10.99$ \\
Group C & $54.32 \pm 5.24$ & $83.12 \pm 5.89$ & $54.65 \pm 7.42$ & $79.75 \pm 3.99$ & $48.76 \pm 6.76$ & $79.43 \pm 6.76$ \\
\hline
\end{tabular}

${ }^{*}$ Compared with groups B and C, $P<0.05 ;{ }^{*}$ compared with group C, $P>0.05$.

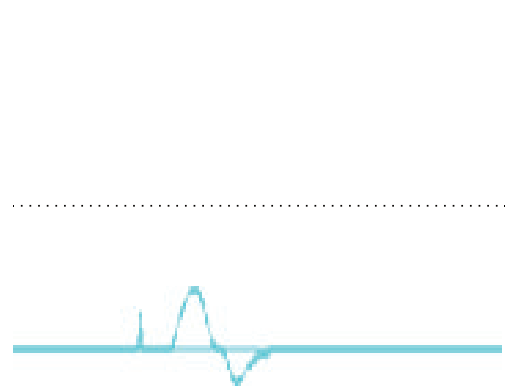

(a)

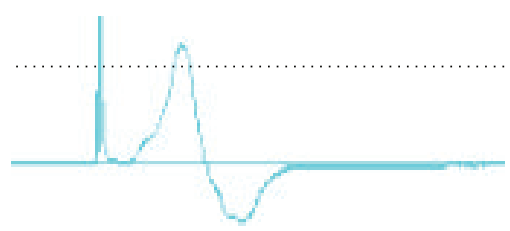

(d)

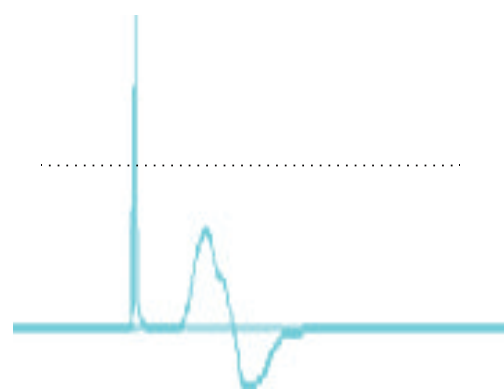

(b)

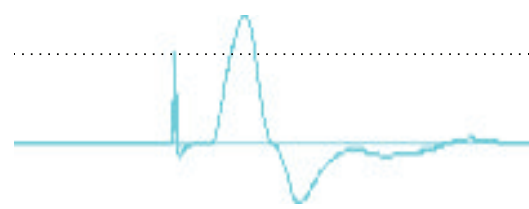

(e)

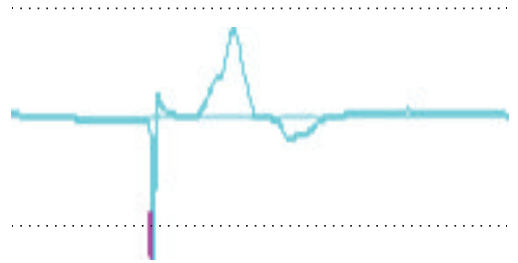

(c)

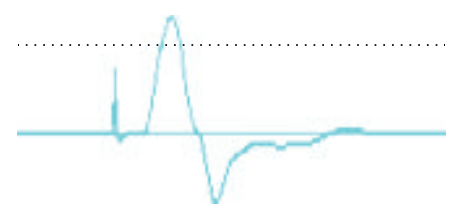

(f)

Figure 3: ((a), (b), and (c)) Neural electrophysiology of group A (a), group B (b), and group C (c) at 6 weeks. ((d), (e), and (f)) Neural electrophysiology of group A (d), group B (e), and group C (f) at 12 weeks.

nerve fibers were sparse; the distribution and diameter of the fibers were irregular, but there were no obvious lymphocyte infiltration (Figures 4(a), 4(b), and 4(c)); $12 \mathrm{w}$ following the surgery, in HE staining slices of three groups, the number of regenerated myelinated nerve fibers in group A was increased; although the myelin was relatively dense, it was irregularly arranged; the number of regenerated myelinated nerve fibers in groups B and C was increased, the myelin was densely and regularly arranged, and there was blood capillary hyperplasia and no obvious lymphocyte infiltration (Figures $4(\mathrm{~d}), 4(\mathrm{e})$, and $4(\mathrm{f}))$.

$6 \mathrm{w}$ following the surgery, in toluidine blue stained slices of three groups, the myelin of regenerated myelinated nerve fibers in group A was sparse, the morphology and diameter in group A were irregular, the myelin of regenerated myelinated nerve fibers in groups $\mathrm{B}$ and $\mathrm{C}$ was dense, but its morphology and diameter were irregular (Figures 4(g), 4(h), and 4(i)); $12 \mathrm{w}$ following the surgery, in toluidine blue stained slices of three groups, there were large numbers of regenerative and partly myelinated axon in group $\mathrm{A}$ and the diameter of them was homogeneous; there were large numbers of regenerative and partly myelinated axon in groups $\mathrm{B}$ and $\mathrm{C}$ and the diameter of them was homogeneous and the myelin sheath of them was more thicker than group A (Figures $4(j)$, $4(\mathrm{k})$, and $4(\mathrm{l})) .6 \mathrm{w}$ and $12 \mathrm{w}$ following the surgery, in stained slices from proximal part of nerve grafts in group $\mathrm{B}$, some blue
BrdU-labeled Schwann cells-like cells' nucleus and brown S100-labeled myelin sheath were observed under microscope (Figures 1(c) and 1(d)).

3.5. Transmission Electron Microscope. $6 \mathrm{w}$ following the surgery, the arrangement and thickness of regenerated myelin sheath in group A were irregular, the arrangement of regenerated myelin sheath in group B was more regular than group A, but the thickness of myelin sheath in group B were not uniform, and the arrangement of regenerated myelin sheath in group $\mathrm{C}$ was more regular than group $\mathrm{A}$ and the thickness of myelin sheath in group $C$ was uniform (Figures 4(m), 4(n), and 4(o)). $12 \mathrm{w}$ following the surgery in group $\mathrm{A}$, the regenerated myelin sheath was thin and irregular, accompanied with Schwann cell proliferation; in group B, the regenerated myelin sheath was denser and thicker, accompanied with Schwann cell proliferation; in group $\mathrm{C}$, the regenerated myelin sheath was denser and thicker than groups A and B, accompanied with Schwann cell proliferation and cytoplasmic organoids such as chondriosome, microtubule, and microfilament inside the nerve (Figures 4(p), 4(q), and 4(r)).

3.6. Recovery Ratio of Quantity, Diameter, and Myelin Sheath Thickness in Regenerated Myelinated Nerve Fibers and Recovery Ratio of Wet Weight of Gastrocnemius Muscle. $6 \mathrm{w}$ and 


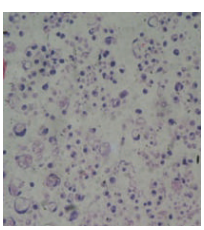

(a)

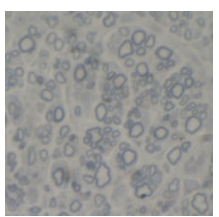

(h)

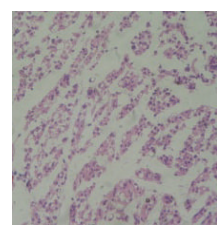

(b)

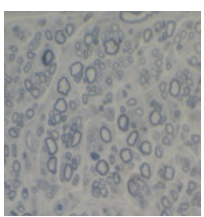

(i)

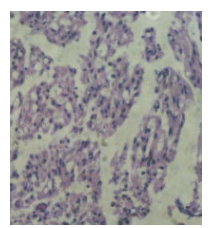

(c)

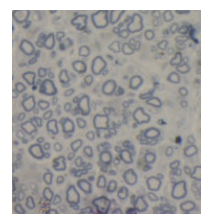

(j)

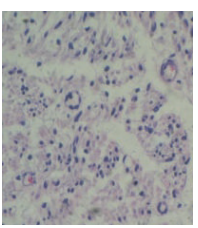

(d)

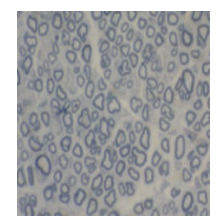

$(\mathrm{k})$

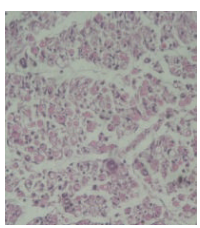

(e)

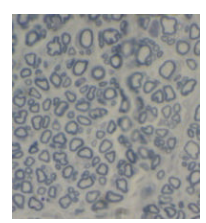

(l)

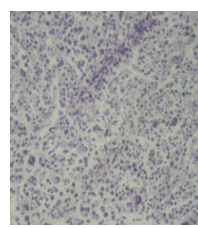

(f)

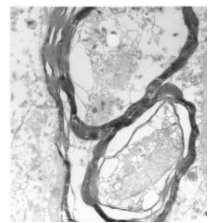

(m)

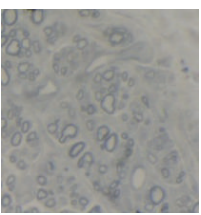

(g)

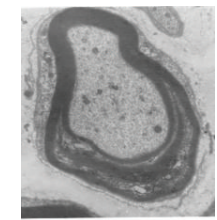

(n)

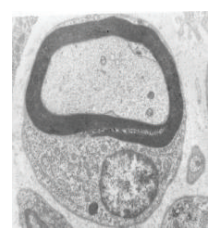

(o)

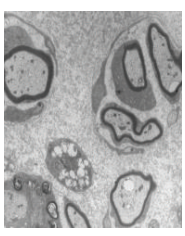

(p)

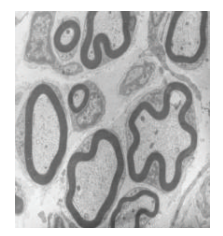

(q)

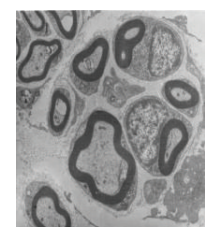

(r)

Figure 4: ((a), (b), and (c)) HE staining of transverse nerve slices from each group at $6 \mathrm{w}$. The regenerated myelinated nerve fibers were sparse; distribution and diameter of the fibers were irregular, but there were no obvious lymphocytic infiltrates. ((d), (e), and (f)) HE staining of transverse nerve slices from each group at $12 \mathrm{w}$. (d) The number of regenerated myelinated nerve fibers in group A was increased; myelin was relatively dense and irregularly arranged. The number of regenerated myelinated nerve fibers in groups B (e) and C (f) was increased; myelin was dense and regularly arranged; there were blood capillary hyperplasia and no obvious lymphocytic infiltrates. ((g), (h), and (i)) Toluidine blue staining slices from each group at $6 \mathrm{w}$. (g) The myelin of regenerated myelinated nerve fibers in group A was sparse; the morphology and diameter were irregular. The myelin of regenerated myelinated nerve fibers in groups B (h) and C (i) was dense, but the morphology and diameter were irregular. $((\mathrm{j}),(\mathrm{k})$, and $(\mathrm{l}))$ Toluidine blue staining slices from each group at $12 \mathrm{w}$. (j) There were large numbers of regenerative and partly myelinated axon in group $\mathrm{A}$ and the diameter of them was homogeneous. There were large numbers of regenerative and partly myelinated axon in groups B (k) and C (l) and the diameter of them was homogeneous and myelin sheath was more thicker than group A. ((m), (n), and (o)) Transmission electron microscope from each group at $6 \mathrm{w} .(\mathrm{m})$ The arrangement and thickness of regenerated myelin sheath in group A were irregular. (n) The arrangement of regenerated myelin sheath in group B was more regular than group A, but the thickness of myelin sheath was nonuniform. (o) The arrangement of regenerated myelin sheath in group C was more regular than group A and the thickness of myelin sheath was uniform. ((p), (q), and (r)) Transmission electron microscope from each group at $12 \mathrm{w}$. (p) The regenerated myelin sheath was thin and irregular, accompanied with Schwann cell proliferation. (q) In group B, the regenerated myelin sheath arranges compactly and thicker, accompanied with Schwann cell proliferation. (r) In group C, the regenerated myelin sheath arranges compactly and thicker than groups A and B, accompanied with Schwann cell proliferation and chondriosome, microtubule, and microfilament inside.

$12 \mathrm{w}$ following the surgery, the recovery ratio of quantity, diameter, and myelin sheath thickness in regenerated myelinated nerve fibers and recovery ratio of wet weight of gastrocnemius muscle of groups $\mathrm{B}$ and $\mathrm{C}$ were superior to those of group A $(P<0.05)$, but the difference between groups $\mathrm{B}$ and $\mathrm{C}$ was not statistically significant $(P>0.05)$ (Table 2).

\section{Discussion}

Schwann cells (SCs) are special gliocytes of peripheral nervous system. They have a close relationship with both the origin, development, morphology, function, and the regeneration of the peripheral nervous system. They provide protection and nutrition and a suitable microenvironment to the axon and promote the formation of myelin sheath. The prerequisite of injured nerve regeneration includes protection of the neurons, induction of axon growth, and guidance of the growth cone to identify the corresponding target organs and establish new functional synapses; Schwann cells play a crucial role in the above three steps [10]. Owing to the limited source and difficulty in cultivation of Schwann cells in vitro and the limited multiplication capacity of mature glial cells, how to acquire enough Schwann cells for transplantation has always been a serious challenge. Adipose derived stem cells (ADSCs) are multipotent stem cells originated from adipose tissue; they possess the capacity to differentiate not only into the traditional mesenchymal cell line $[11,12]$ but also into nonmesenchymal cell types such as skeletal muscle cells, cardiomyocytes, and nerve cells [13-18]. At the same time, ADSCs possess the advantage of extensive sources and superficial location of subcutaneous adipose tissue, and abundant cells are easily obtained from waste adipose tissue (about $1 \times 10^{9}$ stem cell from $10 \mathrm{~mL}$ of adipose tissue) without complicated anesthesia and operation and lower incidence of infection to the donor. In addition, the culture 


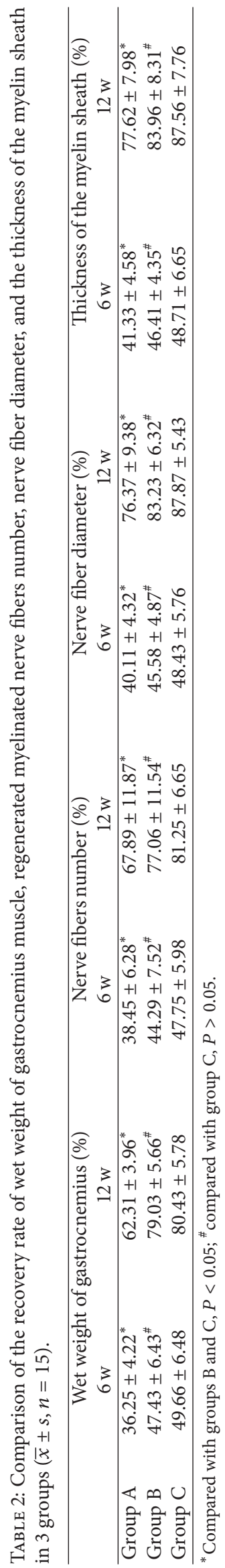


conditions of ADSCs are not strictly required as BMSCs, and the ADSCs possess strong ability in proliferation (average passage time about $60 \mathrm{~h}$ ) and stable multiplication ratio for 13 15 generation; furthermore, the proportion of senile and dead cells during the cell proliferation is low [19], so that ADSCs are gradually becoming an optimum selection for seed cell of tissue engineering.

Researches have shown that allograft nerve that is pretreated by cryopreservation, cryodesiccation, freeze thawing, and chemical process can reduce its antigenicity and avoid immunological rejection after transplantation. The application of acellular nerve allograft that is processed by chemical method in repair of the peripheral nerve defect has been reported by many scholars [3, 20]. Compared with other methods, the components such as cells and myelin which cause immunological rejection during nerve transplantation can be eliminated more thoroughly in acellular nerve allograft that is processed by chemical method, so that the possibility of immunological rejection will be reduced and the histocompatibility will be improved. Furthermore, the integrity of basement membrane which guides the regenerated axons to arrive at the target organs and contribute to the nerve regeneration will be reserved [21]. Thus, the extracellular matrix of acellular nerve allograft is a suitable scaffold that provides an analogical microenvironment for seed cell adhesion, growth, and differentiation into tissueengineering peripheral nerve.

ADSCs can be induced and differentiated into Schwann cells-like cells in vitro $[22,23]$; in this research, the differentiated cell expresses both S-100 and GFAP that exist in Schwann cells. At the same time, it have been observed that ADSCs can adhere and grow in the scaffold when they were implanted into the acellular nerve scaffold; during this period, the cytoactivity of them was similar to the cells in culture media $(P>0.05)$; it means good histocompatibility between cells and acellular nerve scaffold [24, 25]. In this research, when the tissue engineered peripheral nerve that is composed of acellular nerve scaffold and differentiated adipose derived stem cells or only acellular nerve scaffold were implanted to bridge the defect of sciatic nerve, there were no apparent lymphocytes infiltration in the regenerated nerve; it means good histocompatibility between acellular nerve scaffold and allogeneic host rats. $6 \mathrm{w}$ and $12 \mathrm{w}$ following the surgery, there were blue BrdU-labeled Schwann cellslike cells' nucleus and brown S100-labeled myelin sheath was observed in nerve grafts of group B; it means the acellular nerve scaffold provided a suitable living space for cells that were transplanted in vitro; furthermore, these cells have developed into functioning cells in vivo.

\section{Conclusions}

This study shows that tissue engineered peripheral nerve can repair the $10 \mathrm{~mm}$ defect of sciatic nerve effectively and a large number of myelinated nerve fibers pass through the nerve graft $6 \mathrm{w}$ and $12 \mathrm{w}$ following the surgery; it means that the tissue engineered peripheral nerve that composed of acellular nerve scaffold and differentiated adipose derived stem cells have successfully restored the innervation of target organs. The amount of regenerated nerve fibers of group B was obviously more than that of group $A$; the recovery ratio of sciatic nerve function index, wet weight of gastrocnemius muscle, and neural electrophysiology result were superior to those of group A. These results indicate that the recovery index of neural function agrees with the histological results; the probable reason lies in that the differentiation of ADSCs into Schwann cells in vitro increases the amount of Schwann cells, thus accelerating the migration and early regeneration of axons.

\section{Conflict of Interests}

The authors declare that there is no conflict of interests regarding the publication of this paper.

\section{Acknowledgments}

The authors declare that they have no competing interests. They wish to thank all of the authors and their families who so graciously participated in the study. They are indebted to the technicians and staff at the research center of the affiliated tumor hospital of Zhengzhou University.

\section{References}

[1] R. Mohammadi, Z. Esmaeil-Sani, and K. Amini, "Effect of local administration of insulin-like growth factor I combined with inside-out artery graft on peripheral nerveregeneration," Injury, vol. 44, no. 10, pp. 1295-1301, 2013.

[2] A. M. Moore, M. MacEwan, and K. B. Santosa, "Acellular nerve allografts in peripheral nerve regeneration: a comparative study," Muscle \& Nerve, vol. 44, no. 2, pp. 221-234, 2011.

[3] R. J. Nagao, S. Lundy, Z. Z. Khaing, and C. E. Schmidt, "Functional characterization of optimized a cellular peripheral nerve graft in a rat sciatic nerve injury model," Neurological Research, vol. 33, no. 6, pp. 600-608, 2011.

[4] T. W. Hudson, S. Y. Liu, and C. E. Schmidt, "Engineering an improved acellular nerve graft via optimized chemical processing," Tissue Engineering, vol. 10, no. 9-10, pp. 1346-1358, 2004.

[5] Y. Ma, Q. Li, D. Q. Zhao et al., "Isolation, cultivation of adiposederived mesenchymal stem cells and its chondrogenic ability," Xi Bao Yu Fen Zi Mian Yi Xue Za Zhi, vol. 23, no. 5, pp. 463465, 2007.

[6] M. Dezawa, K. Kawana, and E. Adachi-Usami, "The role of Schwann cells during retinal ganglion cell regeneration induced by peripheral nerve transplantation," Investigative Ophthalmology \& Visual Science, vol. 38, no. 7, pp. 1401-1410, 1997.

[7] B. A. Reynolds and S. Weiss, "Generation of neurons and astrocytes from isolated cells of the adult mammalian central nervous system," Science, vol. 255, no. 5052, pp. 1707-1710, 1992.

[8] J. R. Bain, S. E. Mackinnon, and D. A. Hunter, "Functional evaluation of complete sciatic, peroneal, and posterior tibial nerve lesions in the rat," Plastic and Reconstructive Surgery, vol. 83, no. 1, pp. 129-136, 1989.

[9] M. Foidart-Dessalle, A. Dubuisson, A. Lejeune et al., "Sciatic nerve regeneration through venous or nervous grafts in the rat," Experimental Neurology, vol. 148, no. 1, pp. 236-246, 1997. 
[10] R. M. Stassart, R. Fledrich, and V. Velanac, "A role for Schwann cell-derived neuregulin-1 in remyelination," Nature Neuroscience, vol. 16, no. 1, pp. 48-54, 2013.

[11] S. S. Tholpady, A. J. Katz, and R. C. Ogle, "Mesenchymal stem cells from rat visceral fat exhibit multipotential differentiation in vitro," Anatomical Record A Discoveries in Molecular, Cellular, and Evolutionary Biology, vol. 272, no. 1, pp. 398-402, 2003.

[12] S. S. Collawn, N. S. Banerjee, J. de la Torre, L. Vasconez, and L. T. Chow, "Adipose-derived stromal cells accelerate wound healing in an organotypic raft culture model," Annals of Plastic Surgery, vol. 68, no. 5, pp. 501-504, 2012.

[13] A. Cornejo, D. E. Sahar, S. M. Stephenson et al., "Effect of adipose tissue-derived osteogenic and endothelial cells on bone allograft osteogenesis and vascularization in critical-sized calvarial defects," Tissue Engineering A, vol. 18, no. 15-16, pp. 15521561, 2012.

[14] L. Ma, Y. Yang, S. C. Sikka et al., "Adipose tissue-derived stem cell-seeded small intestinal submucosa for tunica albuginea grafting and reconstruction," Proceedings of the National Academy of Sciences of the United States of America, vol. 109, no. 6, pp. 2090-2095, 2012.

[15] M. Jumabay, R. Zhang, Y. Yao, J. I. Goldhaber, and K. I. Boström, "Spontaneously beating cardiomyocytes derived from white mature adipocytes," Cardiovascular Research, vol. 85, no. 1, pp. $17-27,2010$.

[16] R. Madonna, Y. J. Geng, and R. de Caterina, "Adipose cells: characterization and potential for cardiovascular repair," Arteriosclerosis, Thrombosis, and Vascular Biology, vol. 29, no. 11, pp. 1723-1729, 2009.

[17] A. J. Cardozo, D. E. Gómez, and P. F. Argibay, "Neurogenic differentiation of humanadipose-derived stem cells: relevance of different signaling molecules, transcription factors, and key marker genes," Gene, vol. 511, no. 2, pp. 427-436, 2012.

[18] B. Zavan, L. Michelotto, L. Lancerotto et al., "Neural potential of a stem cell population in the adipose and cutaneous tissues," Neurological Research, vol. 32, no. 1, pp. 47-54, 2010.

[19] X. Bai, K. Pinkernell, Y. Song, C. Nabzdyk, J. Reiser, and E. Alt, "Genetically selected stem cells from human adipose tissue express cardiac markers," Biochemical and Biophysical Research Communications, vol. 353, no. 3, pp. 665-671, 2007.

[20] M. Kvist, M. Sondell, M. Kanje, and L. B. Dahlin, "Regeneration in, and properties of, extracted peripheral nerveallografts and xenografts," Journal of Plastic Surgery and Hand Surgery, vol. 45, no. 3, pp. 122-128, 2011.

[21] K. B. Santosa, N. J. Jesuraj, A. Viader et al., "Nerve allografts supplemented with schwann cells overexpressing glial-cell-linederived neurotrophic factor," Muscle \& Nerve, vol. 47, no. 2, pp. 213-223, 2013.

[22] S. Razavi, M. Mardani, M. Kazemi et al., "Effect of leukemia inhibitory factor on the myelinogenic ability of Schwann-like cells induced from human adipose-derived stem cells," Cellular and Molecular Neurobiology, vol. 33, no. 2, pp. 283-289, 2013.

[23] P. Erba, C. Mantovani, D. F. Kalbermatten, G. Pierer, G. Terenghi, and P. J. Kingham, "Regeneration potential and survival of transplanted undifferentiated adipose tissue-derived stem cells in peripheral nerve conduits," Journal of Plastic, Reconstructive \& Aesthetic Surgery, vol. 63, no. 12, pp. e811-e817, 2010.

[24] S. Gao, Y. Zheng, Q. Cai et al., "Comparison of morphology and biocompatibility of acellular nerve scaffolds processed by different chemical methods," Journal of Materials Science: Materials in Medicine, vol. 25, no. 5, pp. 1283-1291, 2014.
[25] G. B. Liu, Y. X. Cheng, and Y. K. Feng, "Adipose-derived stem cells promote peripheralnerve repair," Archives of Medical Science, vol. 7, no. 4, pp. 592-596, 2011. 

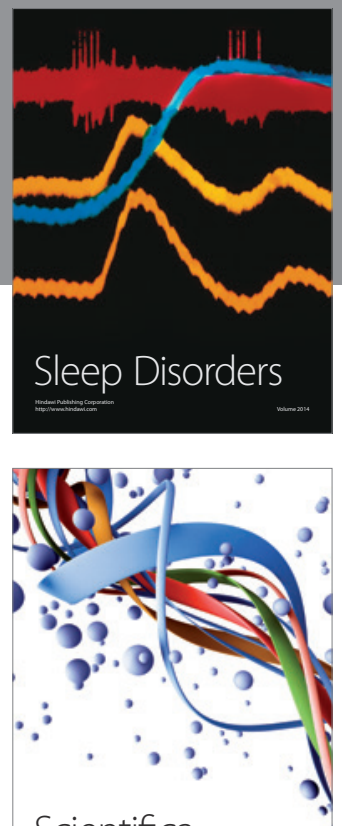

Scientifica
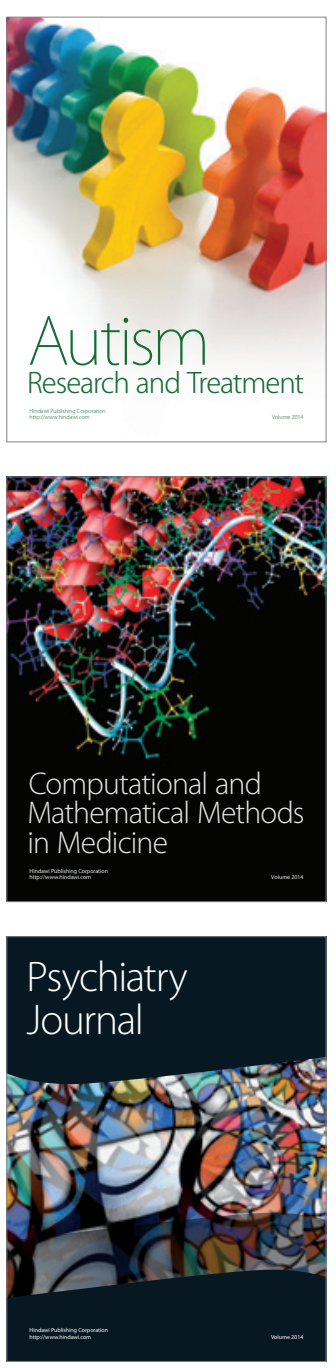
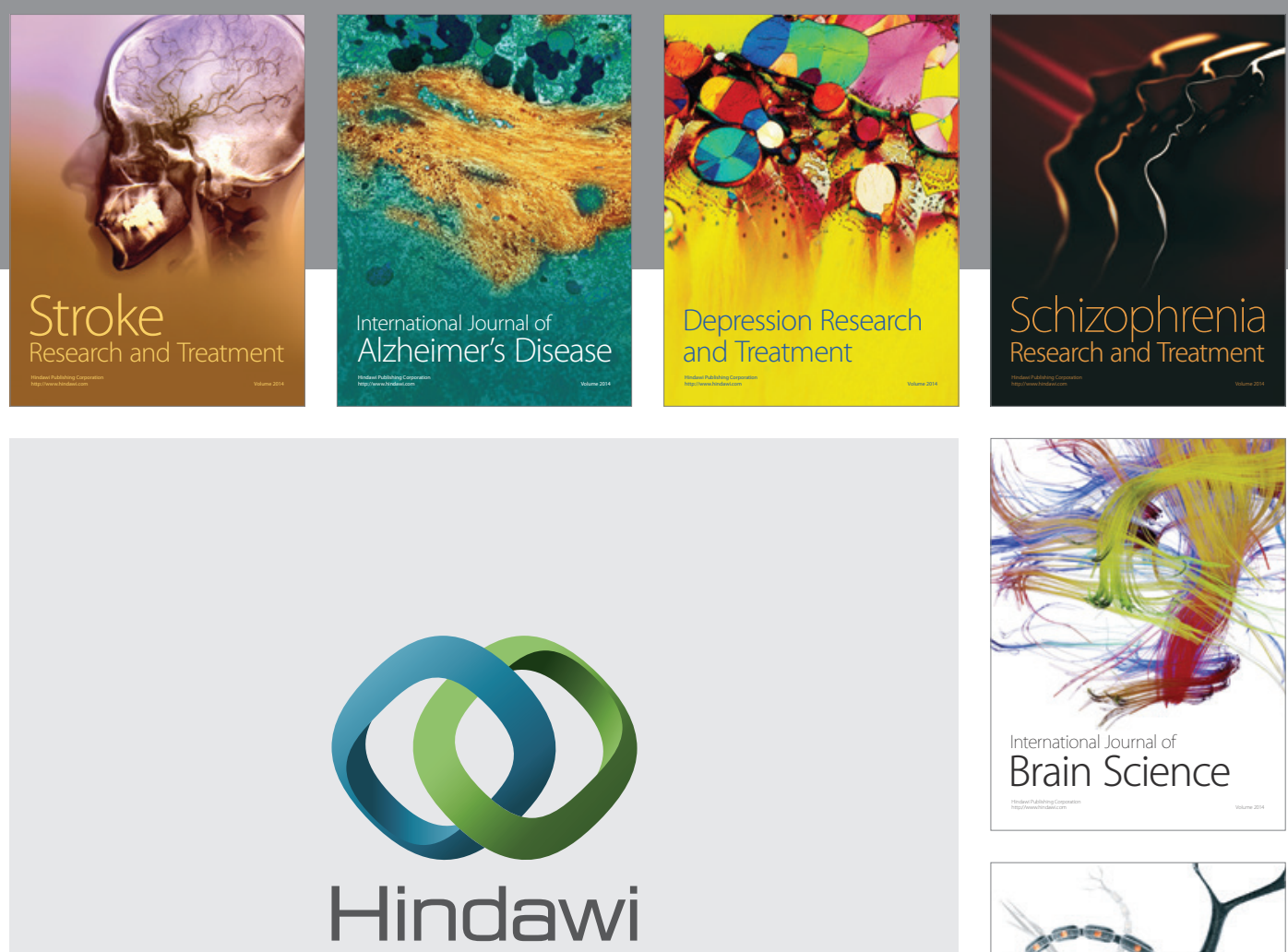

Submit your manuscripts at

http://www.hindawi.com
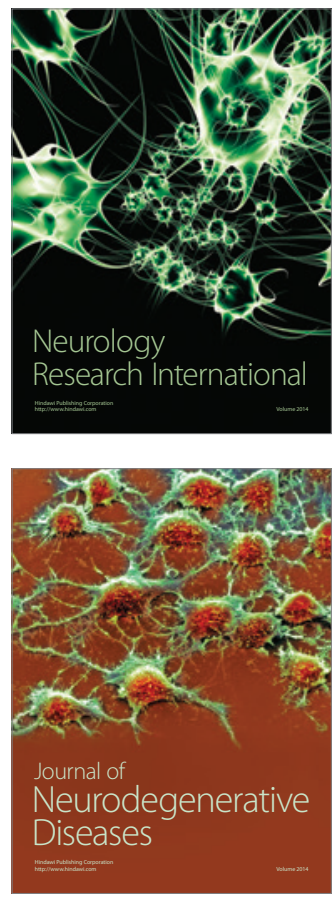

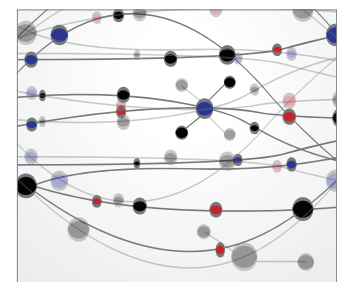

The Scientific World Journal
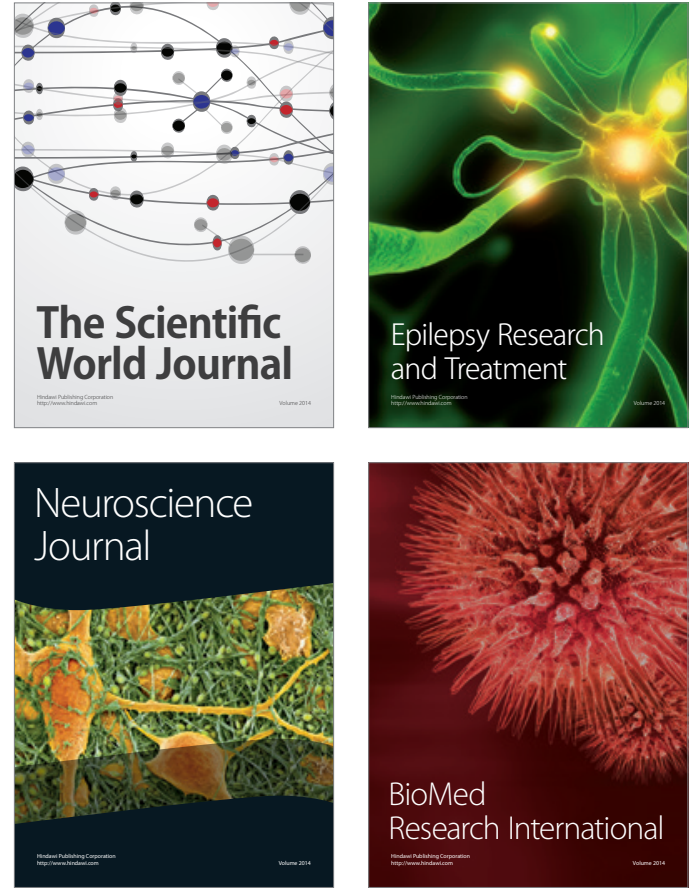

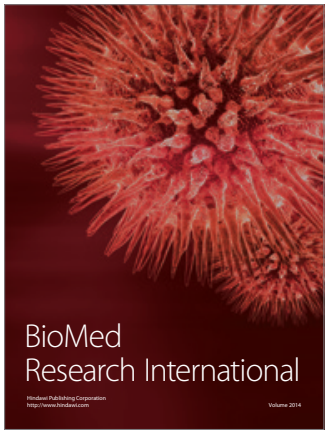

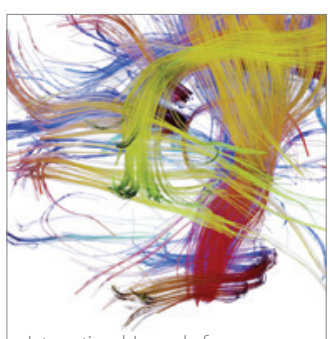

Brain Science

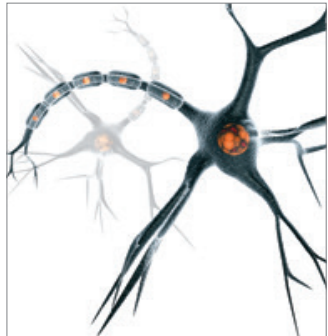

Neural Plasticity
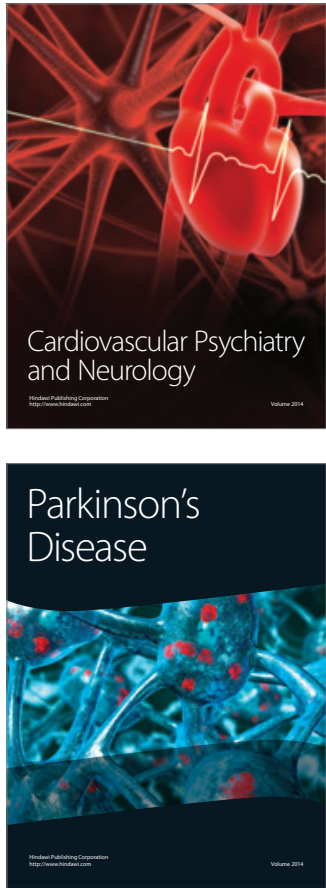